\title{
TÉCNICAS NUCLEARES USADAS NA LOCALIZAÇÃO DE ZONAS DE FRATURAS NOS GRANITOS DE ITU-SP
}

\author{
Francisco Yukio Hiodo' \\ Jorge Luís Porsani² \\ Vagner Roberto Elis ${ }^{3}$ \\ Recebido em 25 de mar., 2002 / Aceito em 22 de nov., 2003 \\ Received Mar. 25, 2002 / Accepted Nov. 22, 2003
}

\section{ABSTRACT}

Nowadays there are many geophysical techniques of rock fractures mapping as GPR, electromagnetic and dipole-dipole geoectrical sounding. These methods don't give information about the content of fracture zone, that can be fullfilled of clay. Using nuclear methods as gamma spectrometry and radon emanometry become possible to identify the nature of mineral composition of fracture material and to study the freshwater circulation using the radon transport by water. In this work the integration of nuclear, GPR and geoelectric techniques in Itu granitic intrusives became very interesting because it was possible to find and identify fracture in granitic rock with clay and freshwater content.

Keywords: Gamma Spectrometry, Radon Emanometry, Granite, Itu, São Paulo.

\section{RESUMO}

Atualmente existem várias técnicas geofísicas de mapeamento de fraturas como GPR, e caminhamentos eletromagnético e geoelétrico dipolo-dipolo. Esses métodos não fornecem informações acerca do conteúdo da zona de fratura, que pode estar preenchida de argila. Usando métodos nucleares, tais como, espectrometria gama e emanomeria de radônio, torna-se possível identificar a natureza da composiç̧ão mineral do material da fratura e estudar a circulaçãa de água usando o transporte de radônio pela água de percolação. Neste trabalho, a integração das técnicas nuclear, GPR e geoelétrica nas rochas intrusivas graníticas de ltu mostrou-se muito interessante porque torna possível encontrar e identificar fraturas em rocha granítica preenchidas com argila ou contendo água doce.

Palavras-chave. Espectrometria gama, GPR, Caminhamento dipolo-dipolo, Granito de Itú-SP, Água em fraturas.

\footnotetext{
IAG/USP. Departamento de Geofísica - Rua do Matão 1226 - Cidade Universitária - São Paulo - SP - 05508-090 - Tel.: (11) 30914777 -Fax: (11) 30915034 E-mail: francisc@iag.usp.br.

2 IAG/USP. Departamento de Geofíisica - Rua do Matão 1226 - Cidade Universitária - São Paulo - SP - 05508-090 - Tel.: (11) 30914734 -Fax: (11) 30915034 E-mail: porsani@iag.usp.br.

3 IAG/USP. Departamento de Geofísica - Rua do Matão 1226 - Cidade Universitária - São Paulo - SP - 05508-090 - Tel.: (11) 30914734 -Fax: (11) 30915034 E-mail: vagnelis@iag.usp.br
} 


\section{INTRODUÇÃO}

Atualmente existem várias técnicas geofísicas de mapeamento de fraturas de rochas preenchidas com algum constituinte mineral. Estes métodos, tais como o radar de penetração no solo (GPR) e os métodos geoelétricos pólo-dipólo, dipólo-dipólo, localizam a fratura com água, mas não trazem nenhuma informação sobre 0 grau de circulação no subsolo, podendo a fratura estar totalmente preenchida com argila, com baixo conteúdo de água livre.

Em muitos casos, as técnicas nucleares podem trazer alguma informação adicional da circulação de água nas zonas de fraturas, apesar da penetração limitada dos fótons gama dos decaimentos radioativos naturais. 0 solo resultante do intemperismo meteórico da rocha do embasamento tem alguma correlação com os minerais presentes no corpo cristalino. Também o gás radioativo $\mathrm{Rn}$ das séries do $\mathrm{U}$ e do Th pode decair antes de escapar para a atmosfera, deixando halos de $\mathrm{P}_{0}$ que podem decair para ${ }^{214} \mathrm{Bi}$ e ${ }^{208} \mathrm{Tl}$, e então ser detectado por espectrometria gama.

\section{METODOLOGIA}

Os métodos nucleares baseiam-se na presença de anomalias de concentração de elementos-filho das séries dos radioelementos naturais ${ }^{238} \mathrm{U}$ e ${ }^{232} \mathrm{Th}$, e também do isótopo natural ${ }^{40} \mathrm{~K}$, ao longo de uma linha cruzando transversalmente a zona de fratura investigada. Outro método usa a emanometria do gás radonio ${ }^{222} \mathrm{Rn}$, de meia vida 3,62 dias, resultante do decaimento alfa do ${ }^{226} \mathrm{Ra}$, da série do ${ }^{238} \mathrm{U}$. A água circulante percola a rocha, arrastando o gás radioativo, que pode percorrer dezenas de metros antes de escapar por alguma fratura, rumo a superfície, mesmo considerando a meia vida pequena deste gás. 0 escape para a atmosfera é proporcionado por um mecanismo de transporte denominado difusão, menos eficiente que 0 arraste.

\section{GEOLOGIA DA ÁREA DE ESTUDO}

A Suíte Granítica de Itu está localizada a aproximadamente 60km da cidade de São Paulo, cobrindo aproximadamente $400 \mathrm{~km}^{2}$. A área compreende uma região de transição entre os terrenos Pré-Cambrianos do Estado de São Paulo e a Bacia Sedimentar do Paraná (PASCHOLATI, 1990).

As rochas encaixantes são principalmente gnáisses com intercalações de xistos, quartzitos, anfibolitos e granulitos pertencentes ao Complexo Piracaia. 0 limite ao Sul da Suíte coincide parcialmente com a Falha de Jundiuvira.
Os contatos são caracterizados principalmente por zonas de cisalhamentos. As estruturas geológicas mais marcantes da Suíte Graníitica de Itu são os lineamentos que correspondem às zonas de falhas curvadas, subparalelas ou convergentes à zona de fal ha de Jundiuvira. Essas zonas coincidem parcialmente com a orientação das principais drenagens da região, como por exemplo os rios Tietê, Piraí e da Fonte.

Os lineamentos retilíneos mais importantes que afetam os corpos granitóides, apresentam orientação segundo os quadrantes NE e NW, onde são abundantes as direções preferenciais de N20-30E e N45-50W. Esses lineamentos representam geologicamente as zonas de fraturas, que são importantes estruturas para a exploração de águas subterrâneas em rochas graníticas.

\section{AQUISIÇÃO DOS DADOS GEOFÍSICOS}

Medidas de espectrometria gama foram feitas com 0 detetor Geofyzika GS512 portátil de Nal(TI) de volume de 0,33 litros, dotado de analisador multicanal de 512 canais. As constantes de calibração do sistema de detecçãa foram obtidas de medidas feitas sobre oito blocos de cimento contendo concentrações conhecidas de radioelementos K,U e Th, do Instituto de Radioproteçũo e Dosimetria -IRD do CNEN. Estes parâmetros permitiram subtrair o ruído de Compton e a interferência entre janelas (stripping), e finalmente converter contagens por minuto obtidas no campo, em valores de concentração dos radioelementos. As medidas foram feitas a cada $5 \mathrm{~m}$ e, os espectros de energia entre 700 $\mathrm{KeV}$ e $3 \mathrm{MeV}$ foram acumulados na memória do detetor. Após tratamento dos dados, onde foram subtraidos o ruído regional e as interferências entre janelas, pôde-se comparar as medidas nas janelas do $\mathrm{K}$, $\mathrm{U}$ e Th.

Para a detecçãa do gás radioativo $\mathrm{Rn}$ são usados tubos de acumulação de PVC de $40 \mathrm{~cm}$ e $10 \mathrm{~cm}$ de diâmetro, estando a extremidade superior selada e a inferior aberta para a entrada do gás contendo ${ }^{222}$ Rn e 0 elemento-filho Po, provenientes do subsolo. Estes tubos são enterrados totalmente no solo por determinado tempo, para a captura do gás antes do escape para a atmosfera. Após completado 0 tempo de acumulação, são feitos dois orifícios na tampa superior para estabelecer um circuito fechado através de um filtro, uma bomba de diafragma de fluxo controlado e um detetor alfacintilométrico de Lucas. Um sistema de circulação forçada de ar de fluxo controlado por um circuito de feedbackfoi desenvolvido para este propósito. 0 ar é arrastado da camara até a célula de Lucas, através da bomba de diafragma de fluxo controlado por um sistema de realimentação formado de um rotâmetro, um dispositivo optoeletrônico infravermelho e um circuito de servocontrole PID, que controla automaticamente o fluxo de um circuito fechado de ar. A medida do Rn é feita por cinco minutos dentro da 
célula de Lucas, em todos os furos para comparação da taxa emanométrica de todos os furos. 0 tempo de acumulação/integração de todas as câmaras é o mesmo, para eliminar-se os efeitos das flutuações de pressão atmosférica.

A taxa de desintegração alfa é medida através de uma célula de Lucas do detetor alfacintilométrico SCINTREX RD-200, de volume 0,10 litros, cuja parede interna é revestida de $\operatorname{ZnS}(\mathrm{Ag})$. Ao interagir com a particula ionizante alfa, este fósforo emite uma radiação luminosa quase na faixa do ultravioleta, que é convertido num pulso elétrico por uma válvula fotomultiplicadora. Um circuito contador controlado por um temporizador ajustável permite determinar o número de eventos ocorridos no intervalo predeterminado.

\section{DISCUSSÃO DOS RESULTADOS}

A área de estudo localiza-se na Fazenda do Chocolate, na borda do batólito de granito de Itu. Naquele local, o granito encontra-se bastante fraturado e a circulação de água é maior em épocas de chuva. Atualmente, com a retirada intensiva de água, muitas dessas fraturas já estão com a circulaçãa de água comprometida. Medidas de emanaçãa de Rn feitas em vários anos consecutivos mostram a mudança das taxas de emanação relacionadas com o problema de recarga das fraturas por águas meteóricas.

Na distância de 50m da linha de espectrometria gama (Figuras 1 e 2) foram identificados claramente picos de concentração de $\mathrm{K}$ e Th, da ordem de duas vezes o nível regional, indicando a presença de uma fratura preenchida com material argiloso. 0 Th - que é insolúvel em $\mathrm{H}_{2} \mathrm{O}$-, após separar-se da rocha, foi transportado até a fratura por modos mecânicos e 0 K, por dissolução. Na ausência de argila, normalmente ocorre empobrecimento de $K$ e pequeno enriquecimento de $\mathrm{U}$, devido a ascensão do gás Rn, cujo elemento-filho ${ }^{214} \mathrm{Bi}$ proporciona contagens na janela usada para detecção do U. Essa anomalia corresponde à zona de reflexão nula (zona de sombra) no radargrama da Figura 4, caracterizada pela presença de material argiloso, que é um excelente atenuador de ondas eletromagnéticas. Esta zona corresponde à região de baixa resistividade elétrica aparente observada no perfil de caminhamento dipolo-dipolo (Figura 5). Também no perfil de espectrometria gama (Figuras 1 e 2) nota-se uma pequena zona de fratura contendo argila, na distância de $34 \mathrm{~m}$, evidenciada pelos picos de Ke Th.

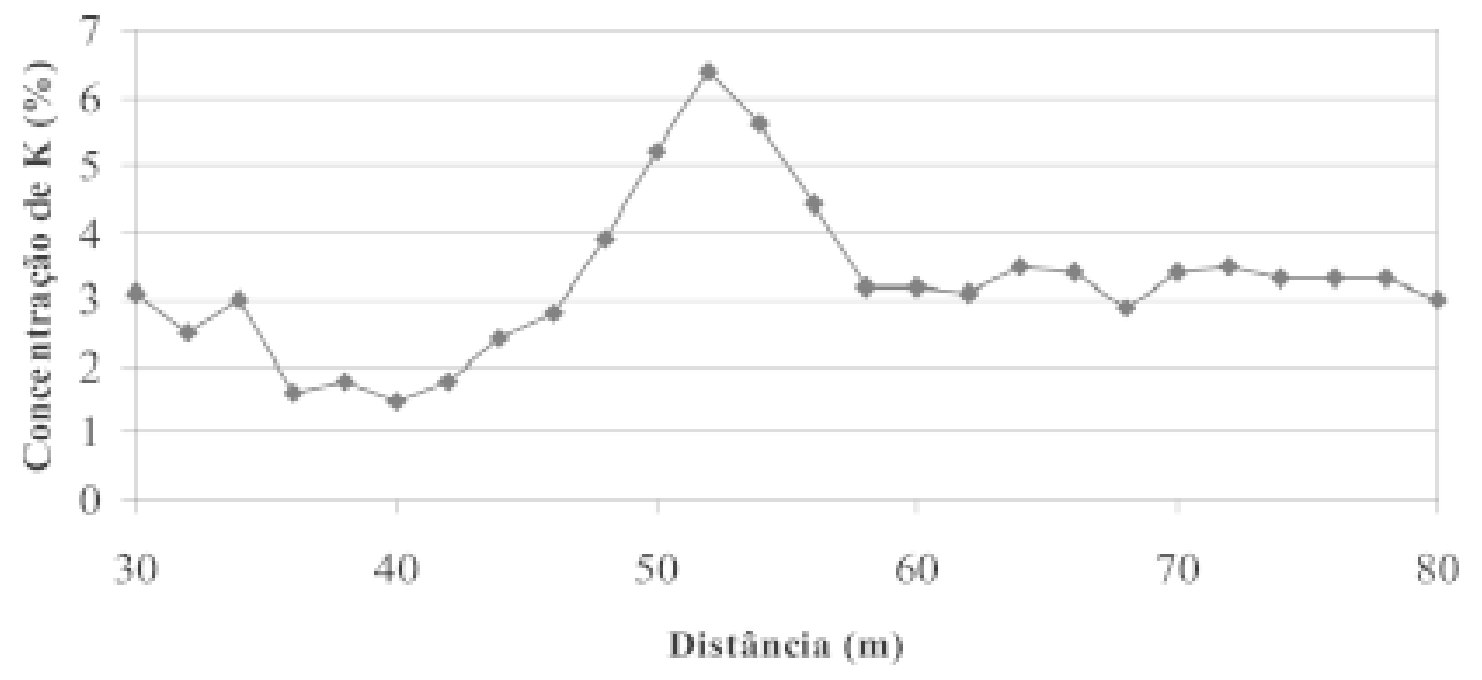

Figura 1 - Perfil gama de concentração de Potássio.

Figure 1 - Gamma profile of potassium concentration. 


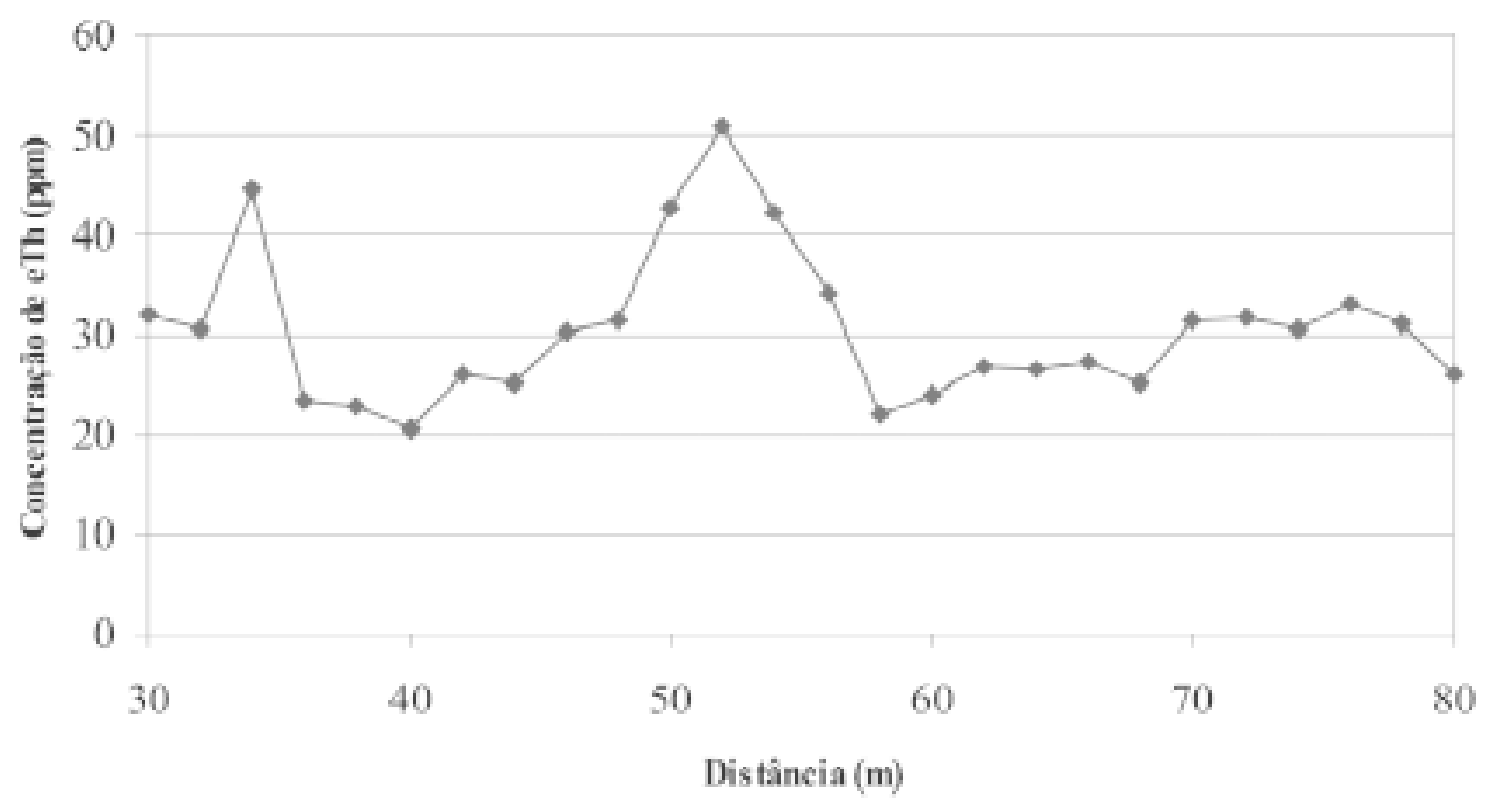

Figura 2 - Perfil gama de concentração de eTh.

Figure 2 - Gamma profile of eTh concentration.

Na Figura 3, obtida por emanometria do gás Rn, nota-se um pico de emanaçã̃o cinco vezes maior que o nivel regional, na distância de $70 \mathrm{~m}$. Nesse mesmo ponto (Figuras 4 e 5), o radargrama e o perfil de caminhamento mostram uma zona muito fraturada, de baixa resistividade elétrica aparente (PORSANI; HIODO; ELIS, 2001). 0 perfil de emanação do Rn permite concluir que as fraturas detectadas podem estar preenchidas com água em circulação.

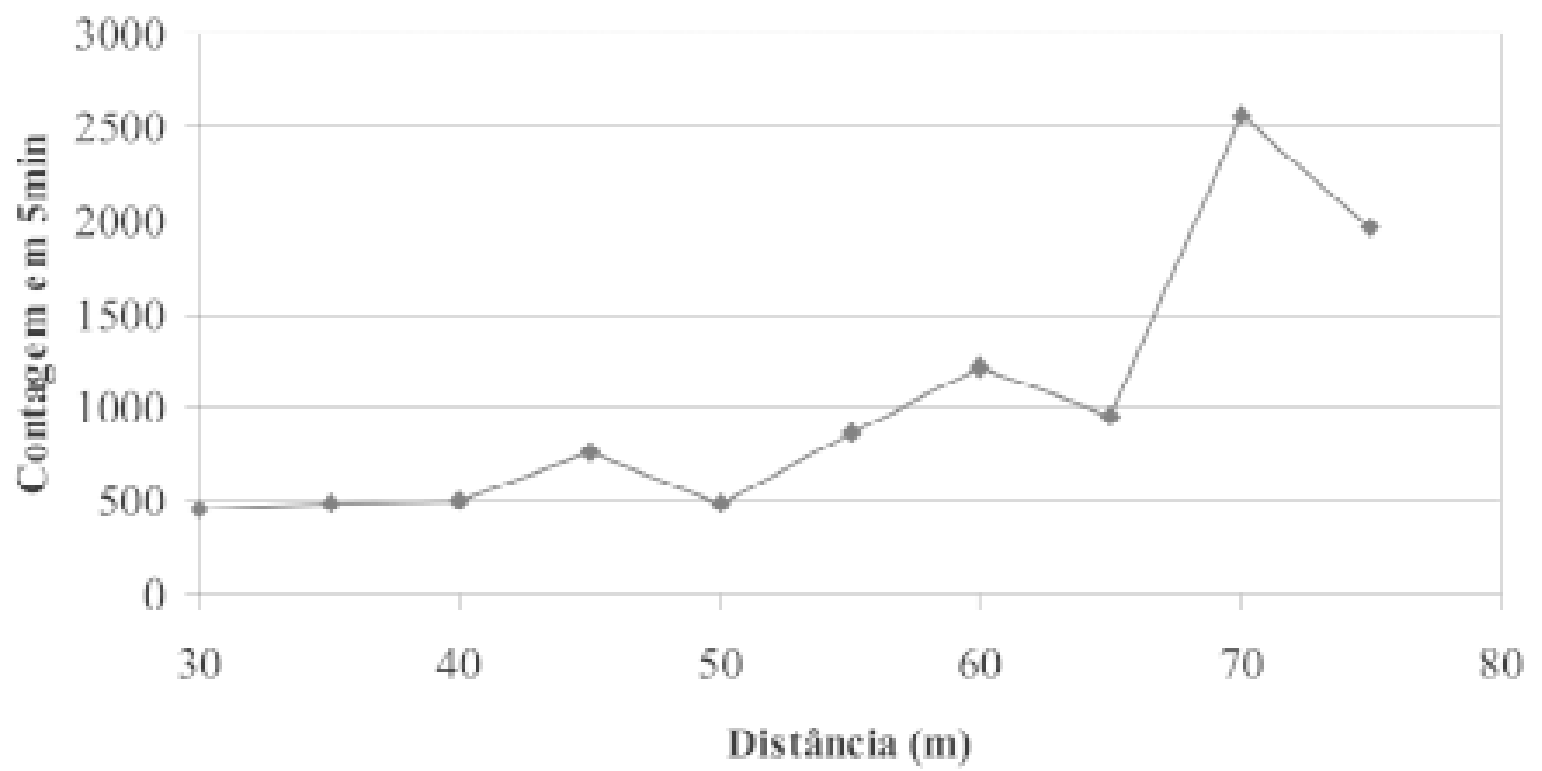

Figura 3 - Perfil de emanação de Radônio.

Figure 3-Emanation profile of radon. 


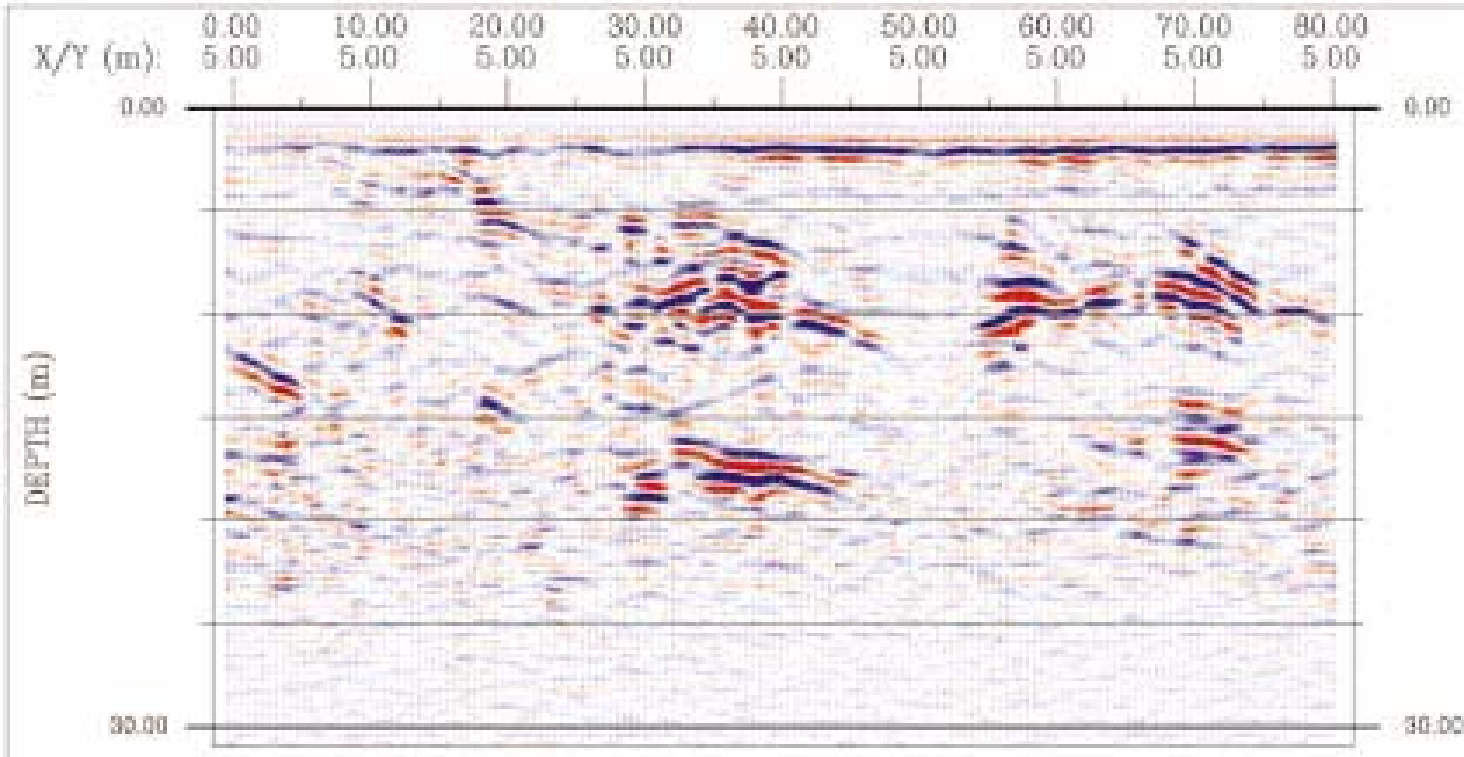

Figura 4 - Perfil de reflexão em profundidade obtido com as antenas de 100MHz. Figure 4 - Profile of reflection in depth obtained by $100 \mathrm{MHz}$ antennas.

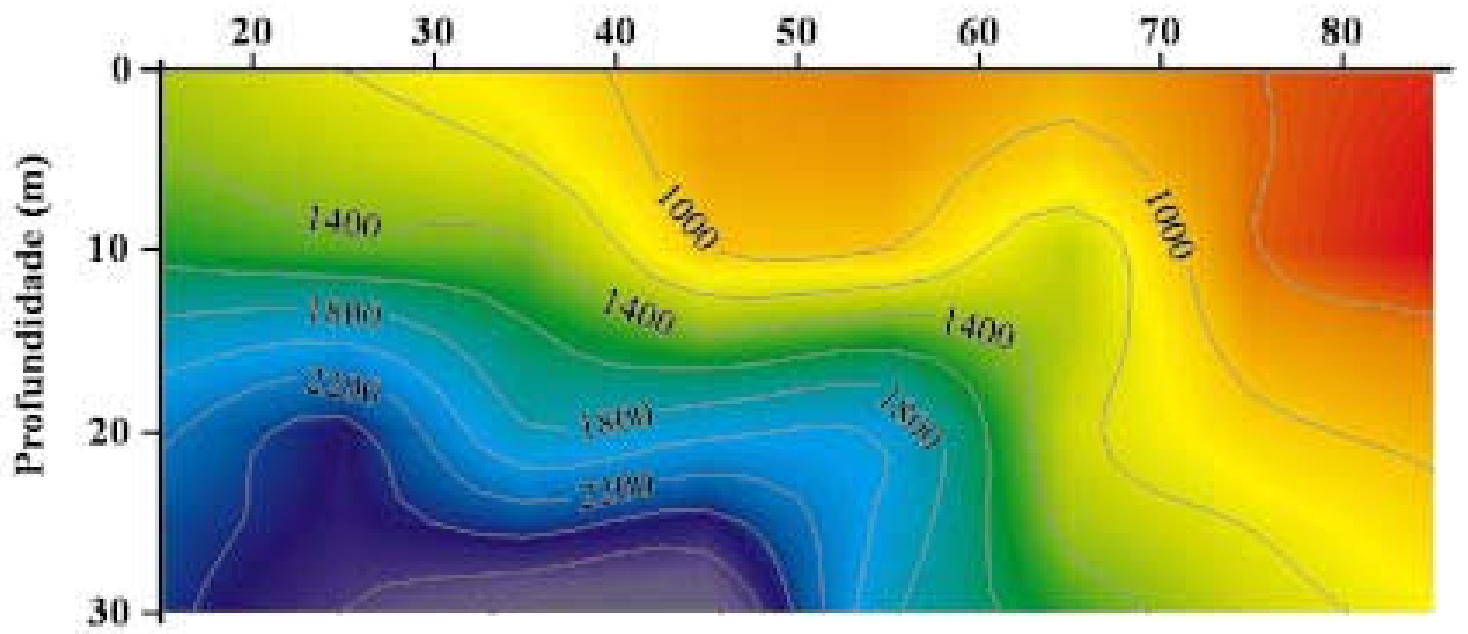

Resistividade Aparente (Ohm.m)

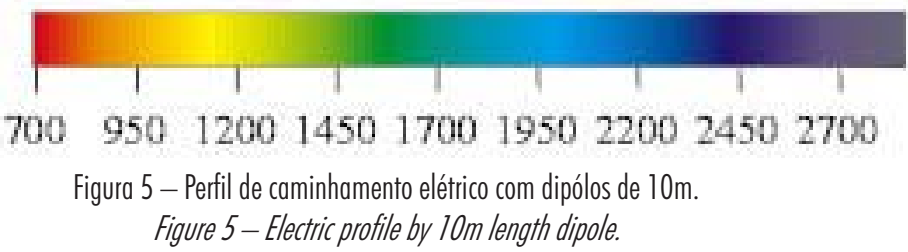




\section{REFERÊNCIAS}

ELIS, V. R. Avaliação da aplicabilidade de métodos elétricos de prospecção geofísica no estudo de áreas utilizadas para disposição de resíduos. 1999. 264 p. Tese (Doutorado)-Instituto de Geociências e Ciências Exatas, Universidade Estadual Paulista, Rio Claro, 1999.

HIODO, F. Y.; MANE, M. A.; YAMABE, T. H. Uso de emanometria do gás radioativo ${ }^{222}$ Rn da série do ${ }^{238} \mathrm{U}$, para detecção de fraturas em rochas.
In: CONGRESSO INTERNACIONAL DA SBGF, 6., 1999, Rio de Janeiro. Anais.. Rio de Janeiro: SBGf, 1999. SBGf 23199.

PASCHOLATI, E. M. Caracterização Geofísica da Suíte Intrusiva de Ittu. 1990. 135 p. Tese (Doutorado)-Instituto Astronômico e Geofísico, Universidade de São Paulo, São Paulo, 1990.

PORSANI, J. L.; HIODO, F. Y; ELLS, V. R. Investigações geofíisicas em rochas graníticas no Omunicípio de Itu, São Paulo-Brasil. In: CONGRESSO INTERNACIONAL DA SBGF, 7., 2001, Salvador. Anais... Salvador: SBGf, 2001.

\section{NOTAS SOBRE OS AUTORES}

Francisco Yukio Hiodo é graduado em Física pelo Instituto de Física da Universidade de São Paulo-USP em 1972. Mestrado em Geofísica pelo Instituto de Astronomia, Geofísica e Ciências Atmosféricas (IAG-USP) em 1981. Doutorado em Geofísica pelo IAG-USP em 1990. Auxiliar de Ensino no IAG-USP em 1973. Professor Assistente em 1981. Professor Doutor do IAG-USP desde 1990. Atualmente é especialista em Instrumentação Geofísica nas áreas de: Paleomagnetísmo (magnetômetros rotativos e desmagnetizadores), Geofísica Nuclear (espectrômetros gama e emanômetros de radônio), Perfilagem de poços tubulares, Geotermometria e sensores de temperatura, Magnetômetros de precessão de prótons e fluxgate, Métodos geoelétricos e eletromagnéticos.

Jorge Luís Porsani é geólogo pelo Instituto de Geociências da UFBa (1987). Mestrado em Geofísica pelo Núcleo de Pesquisas Geofísicas Aplicadas à Prospecção de Hidrocarbonetos da UFPa (1991). De 1991 a 1996, trabalhou como Geofísico no Centro de Pesquisas da PETROBRAS. Doutorado em Geociências e Meio Ambiente pelo Instituto de Geociências e Ciências Exatas da UNESP (1999). Desde 01/12/ 1998 é Docente do Departamento de Geofísica do IAG/USP, atuando com métodos geoelétricos aplicados à geologia, geotecnia, meio ambiente e arqueologia. Atualmente, é Vice Coordenador do Programa de Pós-Graduação em Geofísica e Coordenador do Projeto de Instalação do Sítio Controlado de Geofísica Rasa do IAG (Fapesp 02/07509-1).

Vagner Roberto Elis é graduado em Geologia em 1990 pela Universidade Estadual Paulista. Mestre em Geociências pela Universidade Estadual Paulista em 1993 desenvolvendo a dissertação "A aplicação da geofísica para análise do meio físico: Importância para elaboração de mapeamento geotécnico". Doutor em Geociências pela Universidade Estadual Paulista em 1999 defendendo a tese "Avaliação da aplicabilidade de métodos elétricos de prospecçãa geofísica no estudo de áreas utilizadas para disposição de resíduos". Docente do Departamento de Geofísica do Instituto de Astronomia, Geofísica e Ciências Atmosféricas da Universidade de São Paulo, com ałuação nas áreas de Geofísica Aplicada a estudos ambientais e hidrogeológicos. 